\title{
FISIOLOGIA DO DANO PELO FRIO EM CIRIGUELA (Spondias purpurea L.) ${ }^{1}$
}

\author{
LAESIO PEREIRA MARTINS², SILVANDA DE MELO SILVA³, RICARDO ELESBÃO ALVES ${ }^{4}$, HELOÍSA ALMEIDA CUNHA \\ FILGUEIRAS ${ }^{4}$
}

\begin{abstract}
RESUMO - O objetivo desse estudo foi avaliar a influência do estádio de maturação, temperatura e tempo de exposição na ocorrência de dano pelo frio (DF) em ciriguela. Para a avaliação do estádio de maturação menos suscetível a DF, foram colhidos frutos nos estádios breaker (B), início da pigmentação amarela (IP), amarelo predominante (AP) e expostos a temperaturas de $9,5^{\circ} \mathrm{C}, 10,5^{\circ} \mathrm{C}$ e $14,5^{\circ} \mathrm{C}$, durante $1 ; 3$ e 5 dias. Para a avaliação do desenvolvimento de DF em frutos no estádio AP, foram testadas 10 temperaturas, variando de $14,5^{\circ} \mathrm{C}$ a $5{ }^{\circ} \mathrm{C}$. Os frutos no estádio B apresentaram sintomas irreversíveis de DF a $14,5^{\circ} \mathrm{C}$, após 3 dias, enquanto no estádio IP esses sintomas foram severos, após 5 dias. Para o estádio AP, nenhum sintoma de DF foi verificado entre $9,5^{\circ} \mathrm{C}$ e $14,5^{\circ} \mathrm{C}$. O estádio AP apresentou índice leve de DF a $9,0{ }^{\circ} \mathrm{C}$, após 5 dias. A sensibilidade a baixas temperaturas e a ocorrência de DF em ciriguela foram dependentes do estádio de maturação. $\mathrm{O}$ estádio AP apresentou melhor adaptação a baixas temperaturas, sendo $9,5^{\circ} \mathrm{C}$ a temperatura limite na qual ciriguela pode ser armazenada sem risco de dano pelo frio (DF).
\end{abstract}

Termos para indexação: Estádio de maturação, refrigeração, pós-colheita.

\section{CHILLING INJURY PHYSIOLOGY IN RED MOMBIN FRUIT (Spondias purpurea L.)}

ABSTRACT- The aim of this study was to evaluate the influence of maturation stage, temperature and exposure time on the incidence of chilling injury (CI) in red mombin fruit. For the evaluation of the less susceptible maturation stage to CI, fruits were harvested at breaker (B), pigment initiation (PI), and yellow predominant (YP) stages and exposed to temperature of $9.5^{\circ} \mathrm{C}, 10.5^{\circ} \mathrm{C}$, and $14.5^{\circ} \mathrm{C}$, during 1,3 , and 5 days. For the evaluation of CI development in fruits at YP stage, ten temperatures ranging from $5{ }^{\circ} \mathrm{C}$ to $14.5^{\circ} \mathrm{C}$, were tested. For fruits at $\mathrm{B}$ stage, irreversible $\mathrm{CI}$ symptoms were present at $14.5^{\circ} \mathrm{C}$, after 3 days, while for PI stage these symptoms were severe after 5 days. For the YP stage, no CI symptom was detected between $9.5^{\circ} \mathrm{C}$ and $14.5^{\circ} \mathrm{C}$. The YP stage presented a light $\mathrm{CI}$ index at $9^{\circ} \mathrm{C}$, after 5 days. The low temperature sensitivity and CI occurrence in red mombin fruit were dependent on maturation stage. The YP stage presented the best adaptation to low temperatures and $9.5^{\circ} \mathrm{C}$ was the limit temperature at which red mombin fruit could be stored without CI risk.

Index terms: Maturation stage, refrigeration, postharvest.

\section{INTRODUÇÃO}

A ciriguela é um fruto tropical perecível que se destaca pelo sabor exótico e crescente aceitação no mercado (Sousa et al., 2000; DíazPérez et al., 1998). O armazenamento refrigerado é uma das ferramentas mais importantes utilizadas no prolongamento da vida útil de frutos e hortaliças. Infelizmente, frutos tropicais, a exemplo da ciriguela, e subtropicais são geralmente sensíveis à disfunção fisiológica denominada "chilling injury" ou dano pelo frio (DF) quando mantidos a temperaturas abaixo de um certo limite crítico, acima da temperatura de congelamento, resultando em perdas quantitativas e qualitativas pós-colheita (Wang, 1994). O controle do amadurecimento de um fruto a baixas temperaturas exige o conhecimento dos processos metabólicos característicos deste na época da colheita, bem como das respostas indesejáveis quando este é armazenado sob temperaturas críticas, suscetíveis a DF (Muñoz et al., 2001) que resultam em alterações das características físicas e físico-químicas. Além da temperatura, o tempo de exposição é determinante no desenvolvimento de DF (Wang, 1994).

A incidência de DF provoca o enfraquecimento dos tecidos, tornando-os incapazes de desenvolver normalmente os processos metabólicos, o que é geralmente atribuído à alteração da permeabilidade da membrana lipídica (Nishiba \& Murata, 1996; Lyons, 1973). Os sintomas associados com a ocorrência de dano pelo frio usualmente tornam-se aparentes somente após a transferência do produto para temperaturas mais elevadas (Levitt, 1980; Wang, 1994). No entanto, os tipos, graus e suscetibilidade desses sintomas são variáveis entre tecidos e espécies (Mangrich \& Saltveit, 2000). Os sintomas de DF mais comumente reportados para frutos são a inibição no desenvolvimento e/ou modificação das cores externa e interna dos tecidos, manchas escuras aprofundadas na casca, exsudação, amadurecimento irregular, modificação na textura e no sabor, aumento da incidência da infestação microbiana e aumento da taxa de deterioração (Wang, 1994; Hong et al., 2000; Muñoz et al., 2001).

A temperatura ideal de armazenamento, aquela que potencialmente prolongue a conservação pós-colheita sem promover danos fisiológicos nos frutos, é muito variável (Nishiba \& Murata, 1996). Dessa forma, a cultivar e estádio de maturação são fatores que também interferem nesta variação (Autio \& Bramlage, 1986). Com o objetivo de verificar o estádio de maturação menos suscetível a DF e com melhor potencial de armazenamento, o limite crítico de temperatura para armazenamento e as modificações físicas e físico-químicas associadas com a incidência de DF, esse trabalho visa a avaliar o efeito da redução da temperatura e do estádio de maturação na fisiologia de dano pelo frio em ciriguela.

\section{MATERIAL E MÉTODOS}

Frutos de cirigueleira (Spondias purpurea L.) foram coletados em árvores do pomar do Centro de Formação de Tecnólogos (CFT) da UFPB, situado no município de Bananeiras-PB. Os frutos foram colhidos manualmente no período da manhã, entre janeiro e março de 1999 e 2000, respectivamente. No Laboratório de Biologia e Tecnologia Pós-Colheita do CFT, foram selecionados quanto ao estádio de maturação, ausência de danos mecânicos e doenças aparentes, e sanitizados em solução de 50 ppm de cloro livre. A pesquisa foi desenvolvida em dois experimentos:

Experimento I: Influência do Estádio de Maturação no Desenvolvimento de Danos pelo Frio. Ciriguelas foram colhidas nos estádios de maturação amarelo predominante (AP), início da pigmentação (IP) e "breaker" (B). Os frutos foram mantidos em estufas B.O.D. a $90( \pm 2) \%$ de umidade relativa (UR), em experimentos independentes a $14,5^{\circ} \mathrm{C}, 10,5$ ${ }^{\circ} \mathrm{C}$ e $9,5^{\circ} \mathrm{C}$, até 5 dias, tempo suficiente para ocorrência de DF. Nesse intervalo, os frutos foram transferidos, seguindo-se aos tempos de ex-

\footnotetext{
${ }^{1}$ (Trabalho 196/2001). Recebido: 07/12/2001. Aceito para publicação: 11/12/2002. Parte da Dissertação de Mestrado do primeiro autor. Programa de Pós-Graduação em Agronomia. Centro de Ciências Agrárias (CCA)/Universidade Federal da Paraíba (UFPB). Pesquisa parcialmente financiada pela CAPES.

2 M.Sc., Dept. Tec. Rural (DTR), Centro de Formação de Tecnólogos (CFT)/UFPB, Bananeiras - PB.

${ }_{3}^{3}$ Ph.D., Professor Adjunto III, Lab. Biologia e Tecnologia Pós-Colheita, DCFS/CCA/UFPB, C.P. 04, 58397-970, Areia - PB. E-mail: silvasil@cca.ufpb.br ;

${ }^{4}$ D.Sc., Pós-Colheita, Pesquisador Embrapa Agroindústria Tropical, C.P. 3761, 60511-110, Fortaleza-CE. E-mail: elesbão@ cnpat.embrapa.br; heloisa@cnpat.embrapa.br, respectivamente.
} 
posição de $1 ; 3$ e 5 dias para temperatura ambiente $\left(23 \pm 1^{\circ} \mathrm{Ce} 80 \pm 5 \%\right.$ de UR), onde eram mantidos por 8 horas para estimativa do índice de DF, retornando posteriormente para as respectivas temperaturas de armazenamento. A avaliação subjetiva do índice de DF foi realizada através da escala descrita abaixo. O tempo máximo de exposição de 5 dias foi utilizado com base em pré- experimento realizado para determinar o período máximo onde os sintomas de dano pelo frio desenvolvidos eram mantidos após até 8 horas de permanência dos frutos à temperatura ambiente.

\section{Experimento II: Caracterização do Desenvolvimento de DF de Ciriguelas no EstádioAP}

Ciriguelas colhidas no estádio amarelo predominante (AP) foram submetidas a experimentos independentes conforme Experimento I, a 14,$5 ; 12,5 ; 10,5 ; 10 ; 9,5 ; 9 ; 8,5 ; 8 ; 7$ e $5^{\circ} \mathrm{C}$. Neste experimento, além da avaliação do índice de DF, também foram determinados: firmeza (lb/ $\mathrm{pol}^{2}$ ), com penetrômetro Magness Taylor Pressure Tester, região de inserção de $2 / 16$ polegadas de diâmetro; acidez total titulável (\% de ácido cítrico) e sólidos solúveis totais (\%), segundo Instituto Adolfo Lutz (1985). Estimativa visual de danos pelo frio (DF) foi realizada através da escala: $0=$ não danificado; $1=$ pouco danificado $2=$ danos moderados; 3 = danos severos; 4 = alto grau de danos. O índice de DF foi calculado através de multiplicação do número de frutos em cada categoria pelo respectivo escore, somando o produto e dividindo pelo total de frutos (Kamps et al., 1987; González-Aguilar et al.,1998). O julgamento foi realizado sob luzes fluorescentes por seis avaliadores semitreinados.

$\mathrm{O}$ experimento foi instalado em delineamento inteiramente casualizado, esquema fatorial (10 temperaturas x 3 tempos), com três repetições de 20 frutos cada. As DMSs foram obtidas pelo teste de Tukey, ao nível de pelo menos 5\% de probabilidade.

\section{RESULTADOS E DISCUSSÃO}

Influência do estádio de maturação no desenvolvimento de danos pelo frio.

Os sintomas de danos pelo frio (DF) em ciriguela foram a formação de depressões profundas na superfície dos frutos, necroses nos tecidos, manchas grandes com delineamento de mapas e manchas escuras pequenas, ou 'pitting', inibição do amadurecimento e do desenvolvimento da pigmentação vermelho-escura. Ciriguelas nos estádios B e IP já apresentavam DF após o primeiro dia, a $14,5^{\circ} \mathrm{C}$ (Tabela 1). Mesmo enquanto mantidos a $14,5^{\circ} \mathrm{C}$, a gravidade dos sintomas intensificava-se à medida que o tempo de exposição aumentava, sobretudo para o estádio $\mathrm{B}$. $O$ índice de DF atingido para o estádio B aumentou de 1,0 para 3,0 e para 4,0, após 1 dia de exposição, para ciriguelas mantidas a 14,5; 10,5 e $9,5^{\circ} \mathrm{C}$, respectivamente. Comportamento similar, embora DF presente em menor severidade, foi verificado para o estádio IP. O estádio AP não apresentou nenhum sintoma de DF entre 14,5 a $9,5^{\circ} \mathrm{C}$, durante 5 dias de exposição.

TABELA 1-Danos pelo frio em ciriguelas, avaliadas nos estádios de maturação breaker (B), início do surgimento da pigmentação amarela (IP) e amarelo predominante (AP), em função da temperatura e tempo de exposição (Bananeiras, 2000).

\begin{tabular}{|c|c|c|c|c|}
\hline \multirow{3}{*}{$\begin{array}{c}\text { Temperatura } \\
\left({ }^{\circ} \mathrm{C}\right)\end{array}$} & \multirow{3}{*}{ Tempo de Exposição (dias) } & \multicolumn{3}{|c|}{$\begin{array}{c}\text { Índice de Danos } \\
\text { pelo Frio }\end{array}$} \\
\hline & & \multicolumn{3}{|c|}{ Estádio de Maturação } \\
\hline & & B & IP & AP \\
\hline \multirow{3}{*}{14,5} & 1 & 1,0 & 1,0 & 0 \\
\hline & 3 & 3,5 & 2,2 & 0 \\
\hline & 5 & 3,8 & 2,5 & 0 \\
\hline \multirow{3}{*}{10,5} & 1 & 3,0 & 1,0 & 0 \\
\hline & 3 & 4,0 & 2,5 & 0 \\
\hline & 5 & 4,0 & 4,0 & 0 \\
\hline \multirow{3}{*}{9,5} & 1 & 4,0 & 2,5 & 0 \\
\hline & 3 & 4,0 & 3,0 & 0 \\
\hline & 5 & 4,0 & 4,0 & 0 \\
\hline
\end{tabular}

Escala de 0 a 4 de evolução de danos pelo frio: $0=$ não danificado; $1=$ pouco danificado; $2=$ danos moderados; $3=$ danos severos; $4=$ alto grau de danos.
Os resultados deste experimento indicam que a sensibilidade a DF em ciriguela é função do estádio de maturação, de modo que frutos em estádios mais verdes são mais susceptíveis a DF, como a exemplo do estádio B. A não-manifestação de DF no estádio amarelo predominante (AP), na faixa de 14,5 a $9,5^{\circ} \mathrm{C}$, indica que AP representa estádio de maturidade no qual ciriguelas podem ser armazenadas seguramente, sem risco de DF. O estádio amarelo predominante (AP), portanto, é aquele que possui maior potencial para armazenamento, quando o objetivo é prolongar a vida útil e reduzir as perdas pós-colheita de ciriguelas.

\section{Caracterização de DF em Ciriguelas no Estádio AP.}

No estádio de maturação AP, baixo índice de DF $(0,57)$ foi observado, após 5 dias de exposição a $9^{\circ} \mathrm{C}$ (Tabela 2). Esses índices de DF foram intensificando-se à medida que a temperatura era reduzida e na proporção que o tempo de exposição aumentava. Após 5 dias de exposição, seguido a permanência dos frutos por 8 horas à temperatura ambiente, a ocorrência de DF foi mais acentuada para frutos mantidos a $5^{\circ} \mathrm{C}$. Neste período, os frutos, além dos sintomas de danos pelo frio, apresentavam enrugamento e murchamento.

TABELA 2-Índice de danos pelo frio em ciriguela, no estádio de maturação amarelo predominante (AP), em função da temperatura e do tempo de exposição (Bananeiras, 2000).

\begin{tabular}{cccc}
\hline Temperatura & \multicolumn{3}{c}{ Tempo de Exposição (dias) } \\
\cline { 2 - 4 }${ }^{\circ} \mathrm{C}$ & 1 & 3 & 5 \\
\hline 5 & $1,47 \mathrm{aC}$ & $2,48 \mathrm{aB}$ & $3,93 \mathrm{aA}$ \\
7 & $0,82 \mathrm{bC}$ & $1,55 \mathrm{bB}$ & $2,8 \mathrm{bA}$ \\
8 & $0,37 \mathrm{cC}$ & $0,82 \mathrm{cB}$ & $1,97 \mathrm{cA}$ \\
8,5 & $0,18 \mathrm{cdC}$ & $0,81 \mathrm{cB}$ & $1,08 \mathrm{dA}$ \\
9 & $0 \mathrm{~d}$ & $0 \mathrm{~d}$ & $0,57 \mathrm{eA}$ \\
9,5 & $0 \mathrm{~d}$ & $0 \mathrm{~d}$ & $0 \mathrm{f}$ \\
10 & $0 \mathrm{~d}$ & $0 \mathrm{~d}$ & $0 \mathrm{f}$ \\
10,5 & $0 \mathrm{~d}$ & $0 \mathrm{~d}$ & 0f \\
12,5 & $0 \mathrm{~d}$ & $0 \mathrm{~d}$ & $0 \mathrm{f}$ \\
14,5 & $0 \mathrm{~d}$ & $0 \mathrm{~d}$ & Of \\
\hline
\end{tabular}

Médias seguidas de mesmas letras na vertical (minúsculas) e horizontal (maiúsculas) não diferem entre si, pelo teste de Tukey, a pelo menos 5\% probabilidade.

As disfunções fisiológicas são uma função da temperatura (estresse) e do tempo de exposição (dias ou semanas), podendo resultar no aparecimento de DF caso os frutos sejam mantidos abaixo da temperatura crítica de armazenamento (Levitt, 1980; Kamps et al., 1987). As manchas observadas nos frutos afetados por DF, resposta primária da exposição a baixas temperaturas de resfriamento, são resultantes de eventos relacionados à degeneração dos tecidos (Wang, 1994). Com a elevação do índice de DF, agravavam-se as depressões superficiais nas ciriguelas, provavelmente resultantes de células danificadas que coalesceram, resultando na formação de manchas na forma de mapas, de cor marrom-escura ou com leves tons esverdeados. As pequenas manchas marrons, ou 'pittings', observadas em ciriguelas podem ser resultantes do colapso das células injuriadas, possibilitando rápida invasão de microrganismos deteriorantes (Lyons, 1973). A faixa de temperatura de 8,5 a $5^{\circ} \mathrm{C}$ certamente ultrapassou o limite mínimo de segurança para o armazenamento de ciriguela, não sendo mais possível reverter o nível de dano pelo frio e resgatar o metabolismo normal após transferência desses frutos para temperatura ambiente.

Após 5 dias para frutos mantidos entre 8 e $5{ }^{\circ} \mathrm{C}$, observou-se um decréscimo acentuado na firmeza, comparado ao dia 3 (Tabela 3). Naquelas condições, estes frutos apresentavam-se amolecidos, enrugados, murchos e com alto grau de dano pelo frio. A firmeza dos frutos e hortaliças depende da turgescência, forma e tamanho das células e da composição de substâncias pécticas que compõem a parede celular. A turgescência é produzida pela pressão do conteúdo da célula sobre a parede celular, dependendo das substâncias osmoticamente ativas no vacúolo, da permeabilidade das membranas e elasticidade da parede 
celular (Kays, 1997). A perda acentuada de firmeza observada abaixo de $8,5^{\circ} \mathrm{C}$ pode ser devida à perda excessiva de água quando da exposição do fruto à temperatura ambiente. $\mathrm{O}$ aumento da temperatura resultou num incremento no déficit de pressão de vapor entre os tecidos internos do fruto e o ambiente, resultando na perda de água pelo produto, a qual foi tanto maior quanto mais longo o tempo de exposição a temperaturas mais altas (Levitt, 1980), e quanto maior o nível de estresse causado pelo abaixamento da temperatura (Wang, 1994).

TABELA 3- Firmeza $\left(\mathrm{lb} / \mathrm{pol}^{2}\right)$ de ciriguelas, no estádio de maturação amarelo predominante (AP), em função da temperatura e do tempo de exposição (Bananeiras, 2000).

\begin{tabular}{cccc}
\hline Temperaturas & \multicolumn{3}{c}{ Tempo de Exposição (dias) } \\
\cline { 2 - 4 }${ }^{\circ} \mathrm{C}$ & 1 & 3 & 5 \\
\hline 5 & $2,41 \mathrm{bcA}$ & $1,57 \mathrm{cB}$ & $1,04 \mathrm{dC}$ \\
7 & $2,0 \mathrm{cdA}$ & $1,45 \mathrm{cB}$ & $1,07 \mathrm{cdC}$ \\
8 & $2,4 \mathrm{bcA}$ & $1,48 \mathrm{cB}$ & $1,05 \mathrm{cC}$ \\
8,5 & $2,1 \mathrm{cdA}$ & $1,66 \mathrm{cB}$ & $1,36 \mathrm{bcdC}$ \\
9 & $2,62 \mathrm{abA}$ & $1,47 \mathrm{cB}$ & $1,05 \mathrm{dC}$ \\
9,5 & $2,9 \mathrm{aA}$ & $2,25 \mathrm{aB}$ & $1,40 \mathrm{abcC}$ \\
10 & $2,42 \mathrm{bcA}$ & $1,69 \mathrm{cB}$ & $1,44 \mathrm{abB}$ \\
10,5 & $2,38 \mathrm{bcA}$ & $2,09 \mathrm{abB}$ & $1,63 \mathrm{abC}$ \\
12,5 & $2,11 \mathrm{cdA}$ & $1,76 \mathrm{bcB}$ & $1,66 \mathrm{aB}$ \\
14,5 & $2,42 \mathrm{bcA}$ & $1,57 \mathrm{cB}$ & $1,30 \mathrm{bcdC}$ \\
\hline
\end{tabular}

Médias seguidas de mesmas letras na vertical (minúsculas) e horizontal (maiúsculas) não diferem entre si, pelo teste de Tukey, a pelo menos 5\% probabilidade.

Em geral, observou-se um aumento gradual dos sólidos solúveis totais (SST) à medida que a temperatura e tempo de exposição aumentavam. Após 5 dias de exposição, SST mais altos foram obtidos para frutos mantidos a $12,5^{\circ} \mathrm{C}$ e $14,5^{\circ} \mathrm{C}$. Valores mais baixos de SST foram observados para frutos mantidos a temperaturas inferiores a 9,5 ${ }^{\circ} \mathrm{C}$, sobretudo para aqueles mantidos a $5 ; 7$ e $8^{\circ} \mathrm{C}$, podendo esses serem resultante da inibição do amadurecimento dos frutos (Tabela 4). O aumento dos SST, para frutos mantidos a 12,5 e $14,5^{\circ} \mathrm{C}$, indica que os frutos não sofreram alteração crítica no seu metabolismo, prosseguindo com os processos de maturação e amadurecimento, que resultam na hidrólise do amido em açúcares (Kays, 1997). Por outro lado, este aumento pode, em parte, ser devido à concentração de açúcares e de outros componentes solúveis da célula, devido à perda de água (Muñoz et al., 2001).

TABELA 4-Sólidos solúveis totais (\%) de ciriguelas, no estádio de maturação amarelo predominante (AP), em função da temperatura e do tempo de exposição (Bananeiras, 2000).

\begin{tabular}{cccc}
\hline Temperaturas & \multicolumn{3}{c}{ Tempo de Exposição (dias) } \\
\cline { 2 - 4 }${ }^{\circ} \mathrm{C}$ & 1 & 3 & 5 \\
\hline 5 & $18,40 \mathrm{cB}$ & $19,27 \mathrm{eB}$ & $23,87 \mathrm{bcdA}$ \\
7 & $17,06 \mathrm{dcC}$ & $18,27 \mathrm{eB}$ & $23,20 \mathrm{cdA}$ \\
8 & $17,73 \mathrm{cdB}$ & $18,47 \mathrm{eB}$ & $23,33 \mathrm{cdA}$ \\
8,5 & $18,40 \mathrm{cB}$ & $19,00 \mathrm{eB}$ & $23,00 \mathrm{dA}$ \\
9 & $18,53 \mathrm{bcC}$ & $22,30 \mathrm{bcB}$ & $25,10 \mathrm{bA}$ \\
9,5 & $18,00 \mathrm{cdC}$ & $20,87 \mathrm{cdB}$ & $23,1 \mathrm{cdA}$ \\
10 & $19,00 \mathrm{bcC}$ & $21,20 \mathrm{~dB}$ & $23,33 \mathrm{cdA}$ \\
10,5 & $19,8 \mathrm{bB}$ & $24,40 \mathrm{aA}$ & $24,33 \mathrm{bcA}$ \\
12,5 & $21,87 \mathrm{aC}$ & $23,00 \mathrm{bB}$ & $27,00 \mathrm{aA}$ \\
14,5 & $21,40 \mathrm{aC}$ & $23,13 \mathrm{abB}$ & $25,01 \mathrm{aA}$ \\
\hline
\end{tabular}

Médias seguidas de mesmas letras na vertical (minúsculas) e horizontal (maiúsculas) não diferem entre si, pelo teste de Tukey, a pelo menos $5 \%$ probabilidade.

Tomando-se como referência $9,5^{\circ} \mathrm{C}$, observou-se um decréscimo na acidez total titulável (ATT) em ciriguelas mantidas nas faixas superior e inferior àquela temperatura. Em geral, a ATT também tendeu a diminuir de 1 dia para o $5^{\circ}$ dia de exposição (Tabela 5), sobretudo na faixa de temperatura onde não foi verificado DF, possivelmente resultante do amadurecimento. No entanto, em frutos mantidos a $5{ }^{\circ} \mathrm{C}$, que apresentavam alto grau de DF, também foi observado redução da ATT após 5 dias, como também reportado por Autio e Bramlage (1986) no pericarpo de tomates afetados por DF.

TABELA 5- Acidez total titulável (\% ácido cítrico) de ciriguelas, no estádio de maturação amarelo predominante (AP), em função da temperatura e do tempo de exposição (Bananeiras, 2000).

\begin{tabular}{cccc}
\hline Temperaturas & \multicolumn{3}{c}{ Tempo de Exposição (dias) } \\
\cline { 2 - 4 }${ }^{\circ} \mathrm{C}$ & 1 & 3 & 5 \\
\hline 5 & $0,554 \mathrm{eA}$ & $0,487 \mathrm{~dB}$ & $0,404 \mathrm{fC}$ \\
7 & $0,553 \mathrm{eA}$ & $0,584 \mathrm{abA}$ & $0,556 \mathrm{bcA}$ \\
8 & $0,641 \mathrm{abcA}$ & $0,578 \mathrm{aB}$ & $0,522 \mathrm{cdeC}$ \\
8,5 & $0,632 \mathrm{abcdA}$ & $0,599 \mathrm{aA}$ & $0,538 \mathrm{bcdB}$ \\
9 & $0,671 \mathrm{aA}$ & $0,583 \mathrm{abB}$ & $0,530 \mathrm{cdC}$ \\
9,5 & $0,660 \mathrm{abA}$ & $0,598 \mathrm{aB}$ & $0,504 \mathrm{defC}$ \\
10 & $0,610 \mathrm{bcdA}$ & $0,517 \mathrm{cdB}$ & $0,475 \mathrm{efC}$ \\
10,5 & $0,599 \mathrm{cdeAB}$ & $0,576 \mathrm{cdA}$ & $0,531 \mathrm{cdA}$ \\
12,5 & $0,620 \mathrm{bcdA}$ & $0,525 \mathrm{abB}$ & $0,461 \mathrm{fC}$ \\
14,5 & $0,584 \mathrm{deA}$ & $0,540 \mathrm{bcB}$ & $0,586 \mathrm{abA}$ \\
\hline
\end{tabular}

Médias seguidas de mesmas letras na vertical (minúsculas) e horizontal (maiúsculas) não diferem entre si, pelo teste de Tukey, a pelo menos 5\% probabilidade.

O desenvolvimento da pigmentação vermelho-escura, característica de ciriguelas amadurecidas (Diaz-Pérez et al., 1998; Sousa et al., 2000), foi inibido entre $5 \mathrm{e} 8,5^{\circ} \mathrm{C}$, (mais acentuado para frutos mantidos a 7 e $5^{\circ} \mathrm{C}$ ), concomitantemente à ocorrência de alto grau de DF. A aparência desses frutos foi caracterizada pela predominância da cor amareloopaca e leves tons de vermelho. A inibição do desenvolvimento da pigmentação vermelha também foi verificada na polpa de tomate minimamente processada (Hong et al., 2000) expostos a $5{ }^{\circ} \mathrm{C}$. Esse comportamento indica alteração no processo de amadurecimento desses frutos. Possivelmente, exposição a baixas temperaturas interferiu irreversivelmente na conversão de cloroplastos em cromoplastos (Wang, 1994). O desenvolvimento da cor vermelha, geralmente conferida aos frutos pelos pigmentos carotenóides, antocianinas e licopeno (Kays, 1997), estava precariamente presente em ciriguelas danificadas pelo frio, provavelmente devido à inibição da síntese destes pigmentos. Por outro lado, frutos mantidos acima de $9^{\circ} \mathrm{C}$ desenvolveram normalmente a pigmentação avermelhada após 3 dias de exposição, proporcionando a aparência característica aos frutos.

A exposição a baixas temperaturas pode alterar a distribuição do nível de fosfato na célula, que, conseqüentemente, tem efeito direto no funcionamento das enzimas (Muñoz et al., 2001) que sofrem alterações de suas atividades bem como de sua síntese quando os tecidos são danificados pelo frio (Wang, 1994; Nishiba \& Murata, 1996). Por exemplo, em abobrinha, foi observado aumento da atividade da poligalacturonase (Martínez-Téllez et al., 1998), uma das enzimas responsáveis pelo perda de firmeza dos tecidos (Kays, 1997). As membranas são os locais nas células onde, primariamente, os danos pelo frio ocorrem (Lyons, 1973). Nos tecidos de frutos que manifestam os sintomas de DF, podem ocorrer mudanças estruturais irreversíveis nas camadas lipídicas das membranas (Kamps et al., 1987) e, conseqüentemente, nas proteínas nelas embebidas (Wang, 1994). Estas alterações são dependentes da temperatura e tempo de exposição (Levitt, 1980). Caso o tempo de exposição seja prolongado, isso resultará na perda da integridade e aumento da permeabilidade da membrana, levando a desorganização da estrutura celular, disfunção do metabolismo e acúmulo de substâncias tóxicas, resultando na morte das células (Wang, 1994). Quando o período de exposição ao estresse de baixa temperatura é curto, a transferência dos frutos para temperatura ambiente irá resultar no aumento da atividade metabólica, possibilitando aos tecidos metabolizar o excesso de substâncias tóxicas (Kays, 1997), restaurar seu funcionamento normal, como observado em ciriguelas no estádio AP mantidas sob tempe- 
raturas superiores a $9,5^{\circ} \mathrm{C}$, reparando possíveis danos que possam ter ocorrido nas membranas, organelas ou vias metabólicas. O curto tempo de exposição também possibilitará o restabelecimento de substâncias que foram esgotadas ou impedidas de serem sintetizadas durante o estresse, além do aumento da síntese de ácidos graxos polissaturados, que compõem e protegem as membranas dos danos sofridos durante o curto período de resfriamento (Nishiba e Murata, 1996).

\section{CONCLUSÕES}

Os resultados levam a concluir que: DF em ciriguela é função do estádio de maturação; a temperatura de $9,5^{\circ} \mathrm{C}$ não resultou em $\mathrm{DF}$ em ciriguela no estádio de maturação amarelo predominante (AP); a exposição de ciriguelas no estádio $\mathrm{AP} \mathrm{a} 9^{\circ} \mathrm{C}$ resultou em leve índice de DF após 5 dias de exposição; os sintomas de DF em ciriguela foram o surgimento de manchas marrons e depressões profundas na casca, suspensão do desenvolvimento da cor dos tecidos epidérmicos e inibição do amadurecimento; o estádio de maturação AP é o mais adequado para o armazenamento e aumento da vida útil de ciriguelas; a temperatura de 9,5 ${ }^{\circ} \mathrm{C}$ é a mínima na qual ciriguelas no estádio AP podem ser armazenadas sem risco de danos pelo frio.

\section{REFERÊNCIAS BIBLIOGRÁFICAS}

AUTIO, W.R.; BRAMLAGE, W.J. Chilling sensitivity of tomato fruit in relation to ripening and senescence. Journal of the American Society for Horticultural Science, Alexandria, v.111, n.2, p.201-204, 1986.

DÍAZ-PÉREZ, J.C.; ZAVALETA, R.; BAUTISTA, S.; SEBASTIÁN, V. Cambios físico-químico de ciruela mexicana (Spondias purpurea L.) cosechada en dos diferentes estados de madurez. Revista Iberoamericana Tecnologia Postcosecha, México, v.1, n.1, p.20-25, 1998.

GONZÁLEZ-AGUILAR, G.A.; FORTIZ, J.; BÁEZ-SAÑUDO. Efecto del metil jasmonato sobre la calidade y reduccion de los sintomas de dano por frio en frutos de mango "Tommy atkins". Revista Iberoamericana Tecnologia Postcosecha, México, v.1, n.1, p.32-38, 1998.

HONG, J.H.; MILLS, D.J.; COFFMAN, B.; ANDERSON, J.D.; CAMP, M.J.; GROSS, K.C. Tomato caltivation systems affect subsequent quality of fresh-cut fruit slices. Journal of de American Society for Horticultural Science, Alexandria, v. 126, n.6, p. 729-735, 2000.

INSTITUTO ADOLFO LUTZ. Normas Analíticas do Instituto Adolfo Lutz. São Paulo, 1985, 285p.

KAMPS, T.L.; ISBEIB, T.G.; HERNER, R.C.; SINK, K.C. Evoluation of techiques to measure chilling injury in tomato. HortScience, Alexandria, v.22, n.6, p.1309-1312,1987.

KAYS, S.J. Postharvest Physiology of Perishable Plant Products. Athens:AVI, 1997. $532 \mathrm{p}$.

LEVITT, J. Responses of Plants to Environmental Stresses: chilling, freezing, and high temperature stresses. 2. ed. New York: Academic Press, $1980.497 \mathrm{p}$.

LYONS, J.M. Chilling injury in plants. Annual Review of Plant Physiology, Rockville, v. 24, p.445-466, 1973.

MANGRICH, M.E.; SALTVEIT, M.E. Heat shocks reduce chilling sensitivity of cotton, kenaf, okra, and rice seedling radicles. Journal of the American Society for Horticultural Science, Alexandria, v. 125, n. 3, p. 377-382, 2000.

MARTÍNEZ-TÉLLEZ, M.A. et al. Atividad de poligalacturonasa e firmeza en frutos de calabaza zucchini (Cucurbita pepo L.) almacenados a bajas temperaturas. Revista Iberoamericana Tecnologia Postcosecha, Cidade do México, v.1, n.1, p.70-74, 1998.

MUÑOZ, T.; RUIZ-CABELLO, J.; MOLINA-GARCÍA, A.D.; ESCRIBANO, M.I.; MERODIO, C. Chilling temperature storage changes the inorganic phosphate pool distribuition in cherimoya (Annona cherimola) fruit. Journal of the American Society for Horticultural Science, Alexandria, v. 126, n.1, p. 122-127, 2001.

NISHIBA, I.; MURATA, N. Chilling sensitivity in plants and cyanobacteria: The crucial contribution of membrane lipids. Annual Review of Plant Physiology and Plant Molecular Biology, Rockville, v. 47, p. 541-568, 1996.

SOUSA, R. P.; FILGUEIRAS, H.A.C.; COSTA,J.T.A; ALVES, R.E.; OLIVEIRA, A.C. Armazenamento da ciriguela (Spondia purpurea L.) sob atmosfera modificada e refrigeração. Revista Brasileira de Fruticultura, Jaboticabal-SP, v. 22, n. 3, p. 334-338, 2000.

WANG, C.Y. Chilling injury of tropical horticultural commodities. HortScience, Alexandria, v.29, n.9, p. 986-988, 1994. 